\title{
Numerical Simulation of Impact Tension for Mooring Cable of Underwater Monitoring Platform
}

\author{
Kongde He, Zifan Fang, Yi Zhang, Weihua Yang \\ Mechanic and Material College, China Three Gorges University, Yichang 443002 \\ Email: hekongde@ctgu.edu.cn
}

Keywords: Underwater monitoring platform; Mooring cable; Impact; Numerical simulation

\begin{abstract}
The dynamical model of underwater monitoring platform is formulated aimed at the dynamical response by the action of flow force, based on Hopkinson impact load theory, taken into account the catenoid effect of mooring cable and revised the difference of tension and tangential direction action force by equivalent modulus of elasticity. And solved the equation by hydraulics theory and structural mechanics theory of oceaneering, studied the action force characteristic of mooring cable and motion characteristic of buoy. Through the result the conclusion can be got the buoy will engender biggish heave and swaying displacement, but the swaying displacement got stable quickly and the heaven displacement got vibration for the vortex-induced action by the flow; because the vortex-induced action and the impact action between buoy and the mooring cable, the tension made great changes when the mooring cable was Relaxation-tension.
\end{abstract}

\section{Introduction}

The underwater monitoring platform is a support platform for equipments and instruments of image acquisition for underwater monitoring system, its working environment is complicated and it bears lots of uncertainly loads. Except the gravity is certainly load, the wave load, flow load are uncertainly loads, the randomness of all loads must be considered on design and analysis for buoy. Some scholars were engaged in study the marine mooring platform and submerged floating tunnel and obtained some achievements, this achievements have certain referenced significance for underwater monitoring platform design. Kamman and Huston studied and simulated the variable length and fixed length cable drag system by space discrete method [1], Jutin Mai studied the dynamical response of submerged tunnel under the action of wave flow and reduced the mechanical model to a simply supported beam on two ends, but the model style is not the same as the underwater monitoring platform [4]. The mechanical model of underwater monitoring platform was formed by considering the impact loads and mirroring the Hopkinson impact load theory, solved the equation by hydraulics theory and structural mechanics theory of oceaneering, studied the action force characteristic of mooring cable and motion characteristic of buoy.

\section{Dynamical equation after buoy and mooring cable impact}

The main factors influence buoy moving is the plane motion in the direction of flow velocity, simplified schematic plot is shown as Fig.1.The buoy will emerge instability motion and destroy initial balance when hydrological environment change or meet the extraneous interference. The mooring cable will emerge abrupt tighten or slack by outer interference because it is very light, impact is generated between buoy and mooring cable and impact load is generated too. According to the Hopkinson impact theory[5], the impact force will generate between buoy and mooring cable, the elastic tension will emerge too for the elasticity of mooring cable, at the same time, the buoy receive the drag force along flow direction, resistance of flow, buoyancy and gravity. According to Newton's law of motion, dynamical equations when buoy and mooring cable impact were found as:

$m \frac{d^{2} Y}{d t^{2}}=-A_{c} \rho_{c} C_{0} \frac{d Y}{d t}-k Y-C \frac{d Y}{d t}+F_{Y}$

$$
m \frac{d^{2} Z}{d t^{2}}=-A_{c} \rho_{c} C_{0} \frac{d Z}{d t}-k Z-C \frac{d Z}{d t}+F_{Z}
$$


$m$ is quality of buoy, $A_{c}$ is cross section area of mooring cable, $\rho_{c}$ is density of mooring cable, $C_{o}=\sqrt{E / \rho_{c}}, E$ is modulus of elasticity for mooring cable, $k$ is stiffness coefficient of mooring cable, $C$ is damping coefficient of hydrodynamic force.

The initial displacement and velocity of buoy will emerge after impact, the buoy will move continue according to the initial displacement as starting point, at the end of moving, buoy will get new equilibrium position, according to Newton's law of motion, dynamical equation after buoy and mooring cable impact were found as below:

$$
m \frac{d^{2} Y}{d t^{2}}=-k Y-C \frac{d Y}{d t}+F_{Y} \text { (3) } \quad m \frac{d^{2} Z}{d t^{2}}=-k Z-C \frac{d Z}{d t}+F_{Z}
$$

\section{Static tension and deformation of mooring cable.}

The force diagram of buoy on equilibrium position is shown as Fig.2:

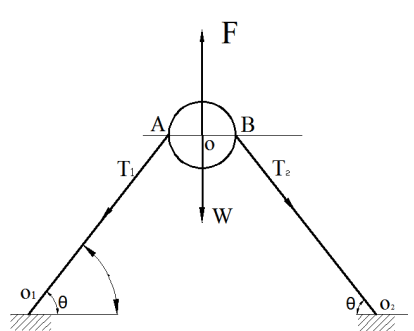

Fig.1 Simplified schematic plot of monitoring platform

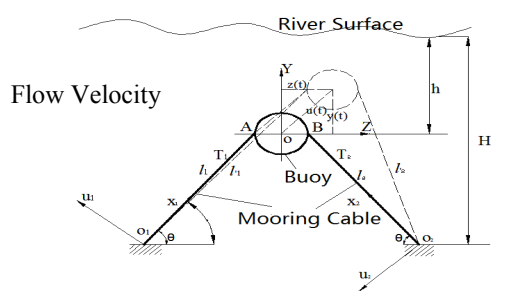

Fig.2 Force diagram of buoy on static balance position

The buoy received the tension of mooring cable $T_{1}, T_{2}$, buoyancy $F$ and gravity $W$ on equilibrium position. The mooring cables were fixed symmetrical, $\theta$ is the fixed angle of mooring cable, according to material mechanics principle, the distortion of mooring cable with the action of $T_{1}, T_{2}$ is: $T_{1}=T_{2}=\frac{F-W}{2 \sin \theta} \quad$ (5) $\quad \Delta l=\frac{T_{1} l}{E A}$

\section{Tension and distortion of mooring cable when buoy move.}

\section{1) Distortion of mooring cable}

The second code on the right of (1),(2),(3),(4)equation is tension of mooring cable for taking the elastic deformation account. The value is product of stiffness and distortion for mooring cable. The distortion for mooring cable on both sides of the buoy is different because the buoy receives the flow force on one side. The elastic distortion of mooring cable for moving is supposed as $u_{i}(t)$, according to fig.3 and deformation compatibility condition of displacement. $u_{1}(t)=l_{1}^{\prime}-l_{1}=Y \cos \theta+Z \sin \theta \quad$ (7) $u_{2}(t)=l_{2}^{\prime}-l_{2}=Y \cos \theta-Z \sin \theta$

\section{2) Stiffness of mooring cable}

The mooring cable is a flexible member which can only receive one-way tensile stress, it will emerge a certain vertical degree by gravity and flow force, so vertical degree can't be omitted when calculating Stiffness of mooring cable. According to Ernst's advice, the deviation can be revised by equivalent elastic modulus method [6], the formula is: $E_{e q}=\frac{E}{1+\frac{\left(q l_{x}\right)^{2} E A_{c}}{12 T^{3}}}$

$q$ is unit length gravity mass of mooring cable, $l_{x}$ is horizontal project length, $T$ is initial tension of mooring cable, according to material mechanics principle, $k=\frac{E_{e q} A_{c}}{l}$.

3) Tension of mooring cable on moving

The force in $\mathrm{Y}$ direction and $\mathrm{Z}$ direction for elastic deformation of mooring cable is below.

$$
k\left[u_{1}(t)+u_{2}(t)\right] \sin \theta=2 k Y \sin \theta \cos \theta \quad(10) \quad k\left[u_{1}(t)+u_{2}(t)\right] \cos \theta=2 k Y \cos ^{2} \theta=2 k Z \frac{\cos ^{3} \theta}{\sin \theta}
$$




\section{Water flow force.}

According to Fig.1, $F_{Y}$ is resultant force for vertical lift force $F_{L}$ of flow and initial force $F_{I Y}$ along $\mathrm{Y}$ direction; $F_{Z}$ is resultant force for drag force $F_{d}$ of flow and initial force $F_{I Z}$ along Z direction; $F_{d}$ can be calculated according to Morison formula.

$$
F_{d}=C_{D} \rho \frac{D}{2}\left|v-\frac{d Z}{d t}\right|\left(v-\frac{d Z}{d t}\right) L(12) \quad F_{I Y}=C_{M} \rho \pi \frac{D^{2}}{4} \frac{d^{2} Y}{d t^{2}} L
$$

$C_{D}$ is viscosity damping coefficient, $\rho$ is density of water, $D$ is diameter of buoy, $v$ is velocity of water, $C_{M}$ is initial force coefficient, $m_{0}$ is unit length mass of buoy, $L$ is length of buoy. $F_{L}$ Can be calculated according to "Karman's vortex" theory:

$$
F_{L}=C_{L} \frac{1}{2} \rho v^{2} D L \quad \text { (14) } \quad F_{I Z}=C_{M} \rho \pi \frac{D^{2}}{4} \frac{d^{2} Z}{d t^{2}} L
$$

\section{Damping coefficient of hydrodynamic force.}

According to reference, damping coefficient of hydrodynamic force $C$ can be calculated according to formula below: $C \approx \frac{4 \omega}{3 \pi} \rho C_{D} A_{L} U$

$\omega$ is undamped nature frequency, $A_{L}$ is cross section area of buoy, $U$ is amplitude of state response for mooring cable.

Dynamical equation after buoy and mooring cable impact.

In respect that mooring cable is three double, put formula (5) (16) into (1), (2), (3), (4), Dynamical equation when buoy and mooring cable impact can be got as below:

$$
\begin{gathered}
\left(m+C_{M} \rho \pi \frac{D^{2}}{4} L\right) \frac{d^{2} Y}{d t^{2}}+\left(6 A_{c} \rho_{c} C_{0}+C L\right) \frac{d Y}{d t}+6 k \sin \theta \cos \theta Y=C_{L} \frac{1}{2} \rho v^{2} D L+(F-G) \\
\left(m+C_{M} \rho \pi \frac{D^{2}}{4} L\right) \frac{d^{2} Z}{d t^{2}}+\left(6 A_{c} \rho_{c} C_{0}+C L\right) \frac{d Z}{d t}+6 k \frac{\cos ^{3} \theta}{\sin \theta} Z=C_{D} \rho \frac{D}{2}\left|v-\frac{d Z}{d t}\right|\left(v-\frac{d Z}{d t}\right) L
\end{gathered}
$$

Dynamical equation after buoy and mooring cable impact can be got as below:

$$
\begin{aligned}
& \left(m+C_{M} \rho \pi \frac{D^{2}}{4} L\right) \frac{d^{2} Y}{d t^{2}}+C L \frac{d Y}{d t}+6 k \sin \theta \cos \theta Y=C_{L} \frac{1}{2} \rho v^{2} D L+(F-G) \\
& \left(m+C_{M} \rho \pi \frac{D^{2}}{4} L\right) \frac{d^{2} Z}{d t^{2}}+C L \frac{d Z}{d t}+6 k \frac{\cos ^{3} \theta}{\sin \theta} Z=C_{D} \rho \frac{D}{2}\left|v-\frac{d Z}{d t}\right|\left(v-\frac{d Z}{d t}\right) L
\end{aligned}
$$

\section{Impact time of buoy and mooring cable}

According to reference, when action time is less than half of natural period of vibration for impacted body, the action load can be called impact load, taking into account the moment of impact, mooring cable will move by buoy action, the period of motion for buoy restrained by mooring cable is $2 \pi \sqrt{\frac{m l}{E A_{c}}}$, so impact time is: $\Delta t=\pi \sqrt{\frac{m l}{E A_{c}}}$

\section{Vertical velocity distribution character of hydrology section for Yangtze River}

The location for fixed underwater monitoring platform was chose Huanglingmiao water area of Yi Chang, according to the statistical data of hydrometric station, the vertical velocity distribution character of the area is accord with Karaueshev's ellipse flow velocity distribution rule. $v=v_{0} \sqrt{1-p \eta^{2}}=\sqrt{p} v_{0} \sqrt{\frac{1}{p}-\eta^{2}}$ 
Where: $v_{0}$-velocity of water surface $(\eta=0) ; p$-distributed parameters of flow velocity, $p=0.6$; $\eta$-relative depth of water from water surface. The vertical mean flow rate $v_{m}$ can be calculated with integration: $v_{m}=\int_{0}^{1} v d \eta=\sqrt{p} v_{0} \int_{0}^{1} \sqrt{\frac{1}{p}-\eta^{2}} d \eta=0.897 v_{0}$

According to fig.3, the velocity of water at the buoy position is: ${ }_{v=\frac{v_{m}}{0.897}} \sqrt{1-0.6\left(\frac{h}{H}\right)^{2}}$

When study the project, hydraulic factors and grain diameters of bed material at the section Huanglinmiao were tested, the test result is shown as Tab.1.

TAB.1 HYDRAULIC FACTORS AND GRAIN DIAMETERS OF BED MATERIAL AT THE SECTION HUANGLINMIAO

\begin{tabular}{|c|c|c|c|c|c|c|c|}
\hline $\begin{array}{l}\text { Number of viewing } \\
\text { perpendicular }\end{array}$ & $1^{\#}$ & $2^{\#}$ & $3^{\#}$ & $\begin{array}{l}\text { Number of viewing } \\
\text { perpendicular }\end{array}$ & $1^{\#}$ & $2^{\#}$ & $3^{\#}$ \\
\hline $\begin{array}{l}\text { Water temperature } \\
\qquad\left({ }^{\circ} \mathrm{C}\right)\end{array}$ & 23.2 & 23.8 & 22.4 & Depth of water(m) & 17.1 & 63.2 & 60.87 \\
\hline $\begin{array}{l}\text { Distance to start } \\
\text { point }(\mathrm{m})\end{array}$ & 510 & 350 & 310 & $\begin{array}{c}\text { Discharge section } \\
\operatorname{area}\left(\mathrm{m}^{2}\right)\end{array}$ & 17922 & 18530 & 17092 \\
\hline $\begin{array}{l}\text { Viewing time } \\
\text { interval (s) }\end{array}$ & 2.27 & 2 & 0.57 & $\begin{array}{l}\text { Width of river } \\
\text { surface }(\mathrm{m})\end{array}$ & 504 & 511 & 495 \\
\hline Rate of flow $\left(\mathrm{m}^{3} / \mathrm{s}\right)$ & 30000 & 42200 & 11200 & $\begin{array}{l}\text { Gradient of water } \\
\text { surface (\%o) }\end{array}$ & 0.102 & 0.181 & 0.019 \\
\hline
\end{tabular}

From TAB.1, the water flow of No. $2^{\#}$ is maximum, water depth is maximum, mean flow rate is $2.27 \mathrm{~m} / \mathrm{s}$, so No. $2^{\#}$ was chose as the fixed position for monitoring platform when study the project.

\section{Numerical example}

\section{Design parameters.}

According to design requirements and above analysis, related parameters are shown as Tab.2.

TAB. 2 DESIGN PARAMETER

\begin{tabular}{|c|c|c|c|}
\hline Length of buoy $(m)$ & 50 & Viscosity resistance coefficient & 0.4 \\
\hline Diameter of buoy $(\mathrm{m})$ & 0.2 & Density of river $\left(\mathrm{kg} / \mathrm{m}^{3}\right)$ & $1 \times 10^{3}$ \\
\hline Diameter of mooring cable $(\mathrm{m})$ & 0.006 & $\begin{array}{l}\text { Mean flow rate of river on buoy } \\
\text { position }_{(m / s)}\end{array}$ & 2.27 \\
\hline Density of mooring cable $\left(\mathrm{kg} / \mathrm{m}^{3}\right)$ & $6.46 \times 10^{3}$ & Lift coefficient & 0.07 \\
\hline $\begin{array}{l}\text { Modulus of elasticity for } \\
\text { mooring cable }(p a)\end{array}$ & $1 \times 10^{11}$ & Mass of buoy $(\mathrm{kg})$ & 1463 \\
\hline Coefficient of initial force & 1 & $\begin{array}{c}\text { Angle of mooring cable and river } \\
\text { bottom }\left({ }^{\circ}\right)\end{array}$ & $21^{\circ}$ \\
\hline Depth of $\operatorname{river}(m)$ & 63.2 & Number of mooring cable & 6 \\
\hline $\begin{array}{l}\text { Distance from buoy to river } \\
\text { surface }(m)\end{array}$ & 10 & Total buoyancy $(N)$ & 17240 \\
\hline
\end{tabular}

\section{Response of buoy.}

Put design parameters into formula (21), impact time can be calculated as: $\Delta t=0.718 \mathrm{~s}$.the velocity curve of $\mathrm{Y}, \mathrm{Z}$ direction were below as Fig.3, Fig.4. Tension graph of mooring cable is shown as Fig.5. 


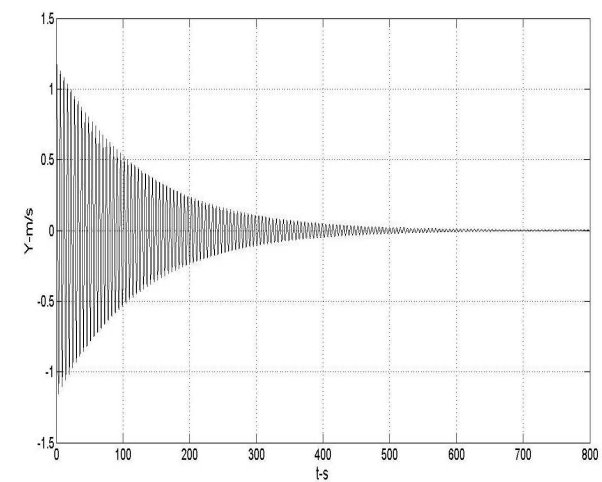

Fig.3 The velocity curve of Y direction

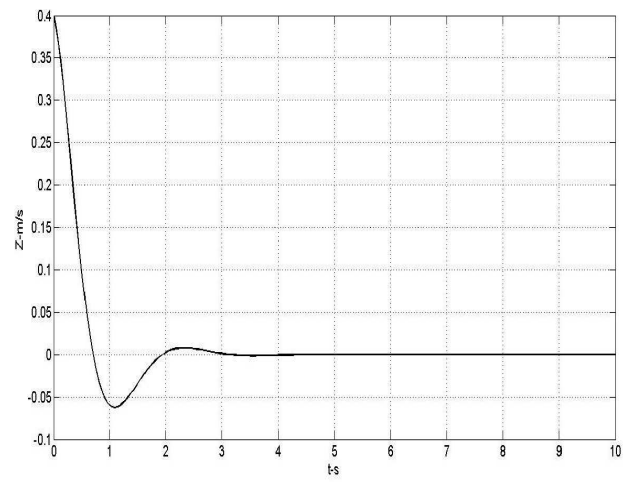

Fig.4 The velocity curve of $Z$ direction

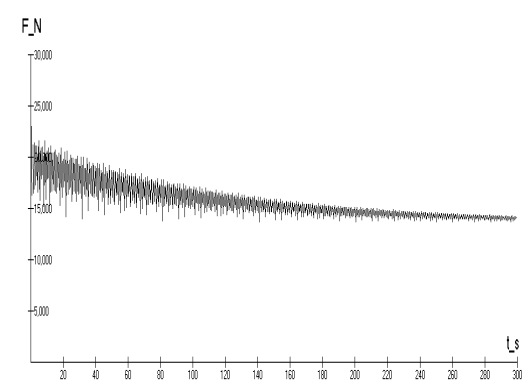

Fig.5 The tension curve of mooring cable

From Fig. 8 conclusion can be got tension of mooring cable is in rise and fall state when buoy moving; at the impact moment, tension get highest, the value is $27000 \mathrm{~N}$, at the stable state tension get $13588 \mathrm{~N}$ and buoy get stability.

\section{Conclusions}

Through the analyze above, the conclusion can be got below: (1) The underwater monitoring platform will emerge biggish sway and heave displacement by drag force, lift force and buoyancy of flow, but along the flow direction buoy get stable quickly and perpendicular to flow buoy vibrate by vortex lift. (2) Because impact exist during mooring cable change from taut to slack state and influence by vortex lift of flow, its tension appears rise and fall state continuously and change greatly. (3) Influence by vortex lift can't be omitted when further study the stability of underwater monitoring platform.

\section{References}

[1] Kamman, James W, Huston, Ronald L, Modeling of variable length towed and tethered cable systems, Journal of Guidance, Control, and Dynamics, 1999, 22 (4) : 602 608.

[2] Jutin Mai. [D]. The Study on Responses of a Submerged Floating Tunnel Subjected to the Wave and Current Chen Du: southwest Jiaotong university, 2005.

[3] Xiaoqin Ma. Impact Dynamics, Press of Beijing Institute of Technology, 1992.

[4] Pao-Hsii Wang and Chiung-Guei Yang. Parametric studies on cable-stayed bridges and structures, 1996,60(2):243-260

[5] Yougang Tang. Structure Mechanics of Oceaneering, Press of Tianjing University, 1992.

[6] Dongyao Wang, Guocan Lin. Vortex-induced nonlinear vibrations of TLP tethersunder circumstances of platform oscillation [J]. Journal of Ocean. 1998, 20(3):119-128. 